\title{
Intelligent Haptic Sensor System for Robotic Manipulation
}

\author{
Pierre Payeur, Member, IEEE, Codrin Pasca, Member, IEEE, Ana-Maria Cretu, Student Member, IEEE, and \\ Emil M. Petriu, Fellow, IEEE
}

\begin{abstract}
Controlling robotic interventions on small devices creates important challenges on the sensing stage as resolution limitations of noncontact sensors are rapidly reached. The integration of haptic sensors to refine information provided by vision sensors appears as a very promising approach in the development of autonomous robotic systems because it reproduces the multiplicity of sensing sources used by humans. This paper discusses an intelligent multimodal sensor system developed to enhance the haptic control of robotic manipulations of small three-dimensional (3-D) objects. The proposed system combines a $16 \times 16$ array of force sensing resistor (FSR) elements to refine 3-D shape measurements in selected areas previously monitored with a laser range finder. Using the integrated technologies, the sensor system is able to recognize small-size objects that cannot be accurately differentiated through range measurements and provides an estimate of the objects orientation. Characteristics of the system are demonstrated in the context of a robotic intervention that requires fine objects to be localized and identified for their shape and orientation.
\end{abstract}

Index Terms-Haptics, laser measurement applications, microprocessors applications, neural networks, object recognition, robot tactile systems.

\section{INTRODUCTION}

C OMPUTER vision and pattern recognition technologies have significantly evolved over the last decade and now appear as standard approaches in numerous robotic applications where objects have to be recognized. Unfortunately, the accuracy that can be reached with these systems remains limited as vision sensors' performance is highly dependent on sophisticated calibration procedures and very sensitive to working environment conditions [1]-[3]. Laser range technologies are now able to provide relatively high resolution under perfect operating conditions [4], [5]. However, their use in industrial environments often results in a significant amount of noise on the data that makes small objects extremely difficult to monitor.

As modern robotic systems tend to reproduce human behaviors in the way they operate, intelligent sensing devices can also take advantage of the duality of sources of information provided by eyesight and touch. It is indeed natural for humans to touch objects in order to get a more precise idea of their shape and texture when visual perception does not provide enough details, like in dark environments or when objects of interest

Manuscript received June 15, 2004; revised April 28, 2005. This work was supported in part by the Natural Sciences and Engineering Research Council (NSERC) of Canada and in part by the Canadian Space Agency.

The authors are with the School of Information Technology and Engineering, University of Ottawa, Ottawa, ON K1N 6N5, Canada.

Digital Object Identifier 10.1109/TIM.2005.851422 are occluded and cannot be directly seen. However, the merge of haptic information with three-dimensional (3-D) models obtained from optical data for robotic manipulation of complex objects has not yet been widely explored in spite of the numerous new possibilities that it opens. As vision-based modeling remains highly sensitive to object's surface reflection characteristics, haptic sensors can be advantageously integrated to complement 3-D models. Following this direction, the addition of a haptic component to visual robotic sensing devices appears as a straightforward improvement that can help in identifying small objects and estimating their characteristics that are critical to achieve haptic control of fine robot manipulations. In this direction, Canepa et al. [6] propose to extend computer vision approaches to tactile data in order to extract cutaneous primitives. Taking advantage of the fact that tactile sensors are directly in contact with the object surface allows to precisely identify fine shape primitives that remain invisible to vision systems.

Recent trends in haptic/tactile sensing focus on the application of this technology in robotics and automation for industrial assembly, assisted surgery where palpation is important [7], or for safe human-robot cooperation [8], [9]. More specifically, a strong interest has been observed in the development of articulated hands made of a few tactile sensitive fingers for dexterous manipulation [10]-[12]. Germagnoli et al. [13] present an approach to drive a robot gripper during the exploration of unknown objects based on the recognition of a limited set of tactile primitives and the interpretation of stress maps with neural networks to locate and follow edges on the object. Pedreno-Molina et al. [14] propose a neural network-based approach that uses artificial skins in guiding grasping operations to ensure stable grasp of object with a two-finger robot hand. Even though relatively good performance can now be achieved with this technology [15], the area and the complexity of the space that can be explored is limited due to the small size of the tactile sensors (mostly a single point) and the fact that they are usually mounted inside the gripper fingertips [16]. Integration of tactile sensors with vision-based approaches allows to circumvent this problem by providing means to select a limited number of areas that need to be touched to refine the resolution of an existing 3-D surface representation.

Through the significant development that occurred on haptic/tactile sensing technologies, the most promising transduction methods and configurations have now been identified [17]. This brings interesting insight in haptic perception for virtual environments representation [18] and 3-D space monitoring necessary for robot movement planning. As haptic perception essentially emulates biological haptic perception mechanisms 
[19], it has been demonstrated that it must integrate two distinct sensing modalities: 1) cutaneous tactile sensing provides information about contact force, contact geometric profile, and eventually the temperature of the touched object [20] and 2) kinesthetic sensing provides information about the position and velocity of the structure carrying the tactile sensor [21].

This paper describes a haptic sensor system that has been developed to be mounted on the end-effector of a serial manipulator arm to refine laser range 3-D maps of workspaces in selected regions where interaction with fine objects is required. In order to provide the two required sensing modalities, a compliant haptic sensing system is proposed that is able to generate an estimate of the object's surface orientation along with a fine description of features located within the sensing area. A multilayer feedforward neural network operating directly on the raw data provided by the haptic sensor allows to achieve an orientation-independent recognition of fine features detected on the surface.

The initial prototype has been developed to operate on a set of features that take the form of geometric symbols defining a pseudorandom encoding scheme that is meant to facilitate the recognition and the localization of each object in a complex scene. Being able not only to model the objects in the scene, but also to precisely segment them in the representation, brings a strategic advantage in guiding the robot during the manipulation phase.

In order to monitor its performance in a realistic industrial context, the proposed sensing system has been mounted on a six degree-of-freedom manipulator and tested for the detection and identification of screw heads having different shapes and orientations. Sections II-V detail all components of the haptic sensor system, the object recognition and orientation estimation procedures using neural networks. Experimental results are also presented on the original pseudorandom set of geometric symbols and with an industrial testbed where a robot is guided by a laser range finder to determine regions that must be touched to determine the type and orientation of screw heads that need to be removed.

\section{Kinesthetic Sensing System}

The proposed robotic haptic sensing system consists of a custom-designed instrumented passive-compliant wrist and a tactile array sensor [21] mounted on a commercial manipulator as shown in Fig. 1. The tactile sensor being a sensitive device with a very limited range of displacement (within a millimeter), mounting it directly on the robot end-effector would make the cutaneous measurements highly dependent on the position and orientation of the robot arm. Forces applied by the robot arm on the object, and the tactile sensor, would influence the cutaneous measurements as the deformation of the tactile array varies according to the position of the end-effector along the normal to the surface. A complex compensation scheme would then be required to ensure consistency of the measurements. Moreover, measuring directly with the tactile probe would imply that the orientation of the robot end-effector is maintained perfectly perpendicular to the object surface while cutaneous information are measured. As such a specific orientation is

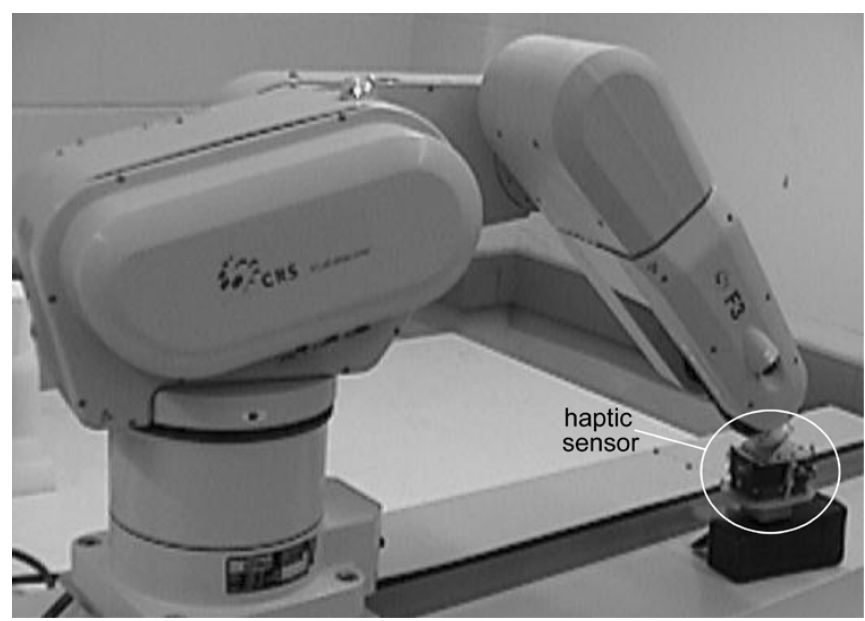

Fig. 1. Haptic perception system mounted at the end of the serial robot manipulator.

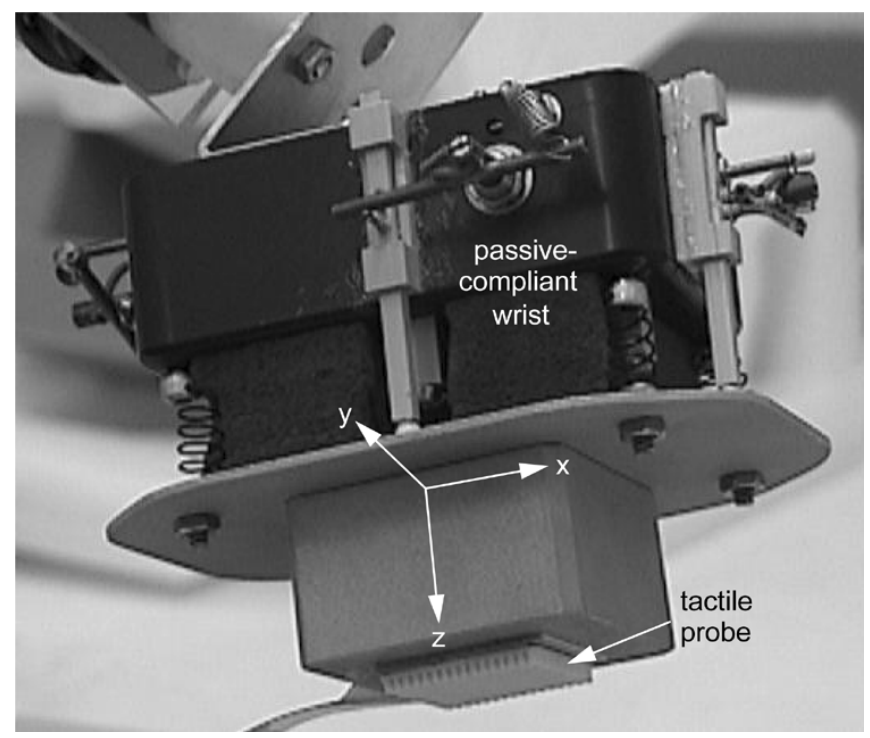

Fig. 2. Instrumented passive-compliant wrist with the tactile sensor probe.

virtually impossible to achieve in environments where the structure of the scene is not known a priori, a special device must be added between the robot end-effector and the tactile sensor to compensate for misalignments. This device can also be designed to ensure uniform forces distribution on the tactile probe, reducing the sensitivity to robot arm position.

The compliant wrist included in the proposed haptic sensor system allows the tactile sensor to accommodate the constraints of the local geometry of the explored object surface and, thus, to increase the amount of information acquired. The instrumented passive-compliant wrist shown in Fig. 2 consists of two rigid planar plates, one being passively mounted on springs. This provides the wrist with three relative degrees of freedom: two rotations about the $x$ and $y$ axes of the tactile probe's plane and one displacement along the $z$ axis (normal to the tactile sensor's plane). Four linear displacement transducers allow one to measure the distances between the two plates of the wrist in four positions, enabling the calculation of the relative orientation and distance between the wrist's plates. Referring to the base of the robot, position sensors placed in the robot's joints along 
with the coordinates provided by the instrumented passive-compliant wrist determine the kinesthetic information about the tactile sensor position and orientation.

In order to collect tactile data in phase with position and orientation measurements provided by the passive-compliant wrist, an onboard electronic circuit based on PIC microcontroller technology has been designed to gather the data from the four linear displacement transducers of the compliant wrist and to calculate the $4 \times 4$ homogeneous transformation matrix representing the kinesthetic sensor data that define the tactile sensor position and orientation with respect to the robot arm. The microcontroller also provides a serial data communication interface to transfer these parameters to the user interface.

\section{TACTILE SENSOR}

The tactile sensor performs the cutaneous component of sensing that provides the contact geometric profile of the touched object. This information being combined with that of the passive-compliant wrist allows a complete reconstruction of the 3-D shape of the surface located within the area of the tactile sensor probe.

\section{A. Tactile Sensor Transducer}

The most popular tactile sensor technologies use conductive elastomer, piezoelectric effects, or piezoresistive properties to measure a contact force profile rather than a contact displacement profile. Under the pressure from an external bias force, the local object geometric profile indents an elastic pad [17]. The induced contact forces are transmitted through an elastic overlay to a force sensitive array of transducers. The measurements provide a local 3-D geometric profile (shape) of the touched object surface. The elastic pad acts as a low-pass filter and displacement-to-force converter, while the force sensor array accomplishes a two-dimensional (2-D) sampling and a one-dimensional force sensing. The principle of operation is illustrated in Fig. 3(a). This results in a nonlinear relationship between the displacement and the measured strain in the elastic pad [22], [23] which depends on the properties of the elastic overlay. Such a force sensitive tactile sensor provides a very robust construction which is suitable for industrial applications.

In the current research work, a piezoresistive tactile sensor has been built from a commercially available matrix of force sensing resistor (FSR) elements on which an elastic overlay was added to allow contact with nonuniform surfaces over the entire tactile probe surface. The array of FSR elements consist of a $16 \times 16$ matrix of pressure-sensing points respectively spaced by $1.5875 \mathrm{~mm}$ (1/ $16 \mathrm{in})$ and uniformly spread on a $645.16 \mathrm{~mm}^{2}$ (1 square inch) area as shown in Fig. 4(a). ${ }^{1}$ The FSR elements exhibit exponentially decreasing electrical resistance with applied normal force. The relationship between the applied force $F$ and the resistance $R$ of the FSR is of the form

$$
F \propto \frac{1}{R^{\beta}}
$$

where $\beta$ is sensor dependent and is estimated by calibration. This relationship holds over the operational range of the sensor

${ }^{1}$ Force Sensing Resistors (FSR). Interlink Electronics Inc. Camarillo, CA, 2002; http://www.interlinkelec.com.

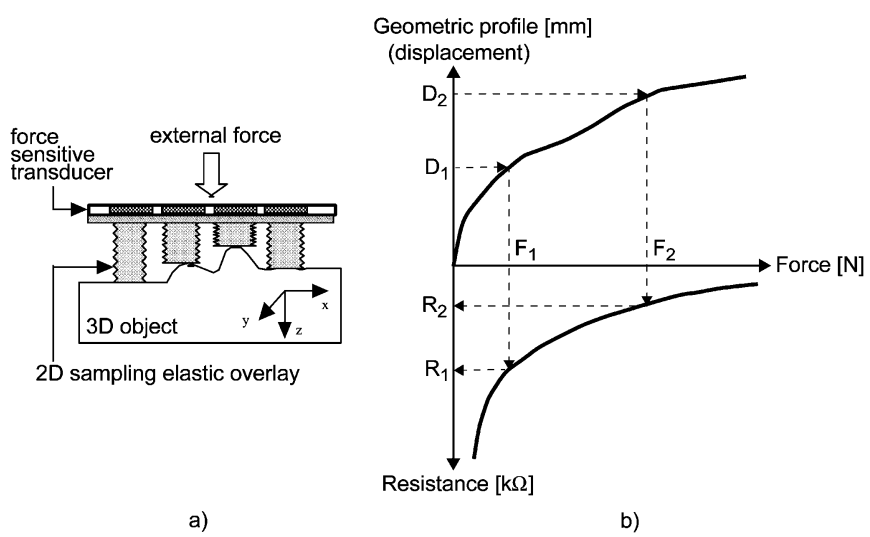

Fig. 3. Force/displacement/resistance characteristics of the force-sensitive transducer with (a) its elastic overlay and (b) the relationship with the displacement produced by the shape of the probed object.

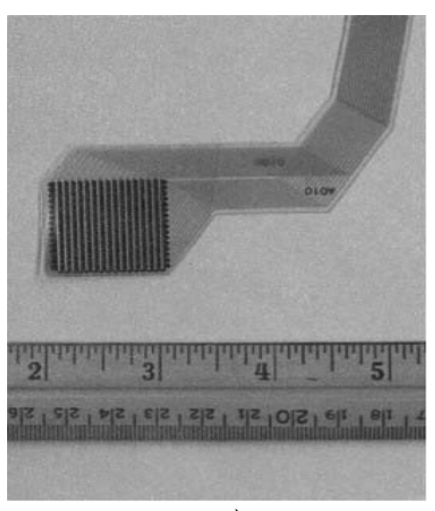

a)

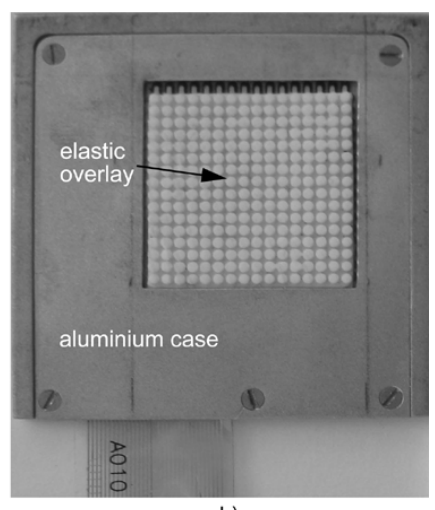

b)
Fig. 4. (a) Force-sensing resistor array covered by (b) the $16 \times 16$ tab-shaped elastic overlay mounted in its protective aluminum case.

beyond which saturation is observed making the resistance remain constant in spite of further increase in the force applied as shown in Fig. 3(b). For the sensor used in this experimentation, the pressure range varies within 1 to $100 \mathrm{~N} / \mathrm{cm}^{2}$ while the resistance value changes from $200 \Omega$ up to a stand-off value in the Megohm range.

The FSR elements sense compression forces and, thus, must be placed on a rigid backing provided by an aluminum protective case, shown in Fig. 4(b), which is mounted on the second planar plate of the passive compliant wrist. The elasticity of the overlay resets the tactile measuring system when the sensor ceases to touch the object. Moreover, it limits the force applied on the FSR array through a damping effect, thus, ensuring physical protection of the FSR elements against impulsive contact forces and preventing electrical saturation of the device [24], [25].

The elastic overlay installed on the top of the FSR array is made of a proprietary elastic pad with protruding round tabs sitting on top of each node of the FSR matrix providing a de facto uniform spatial sampling as shown in Fig. 4(b). This configuration avoids the inherent blurring effect that occurs when onepiece elastic pads are used. Based on recommendations made in [26], [27], circular tabs have been designed to occupy $50 \%$ of each 2-D sampling area. This allows each tab to expand laterally 


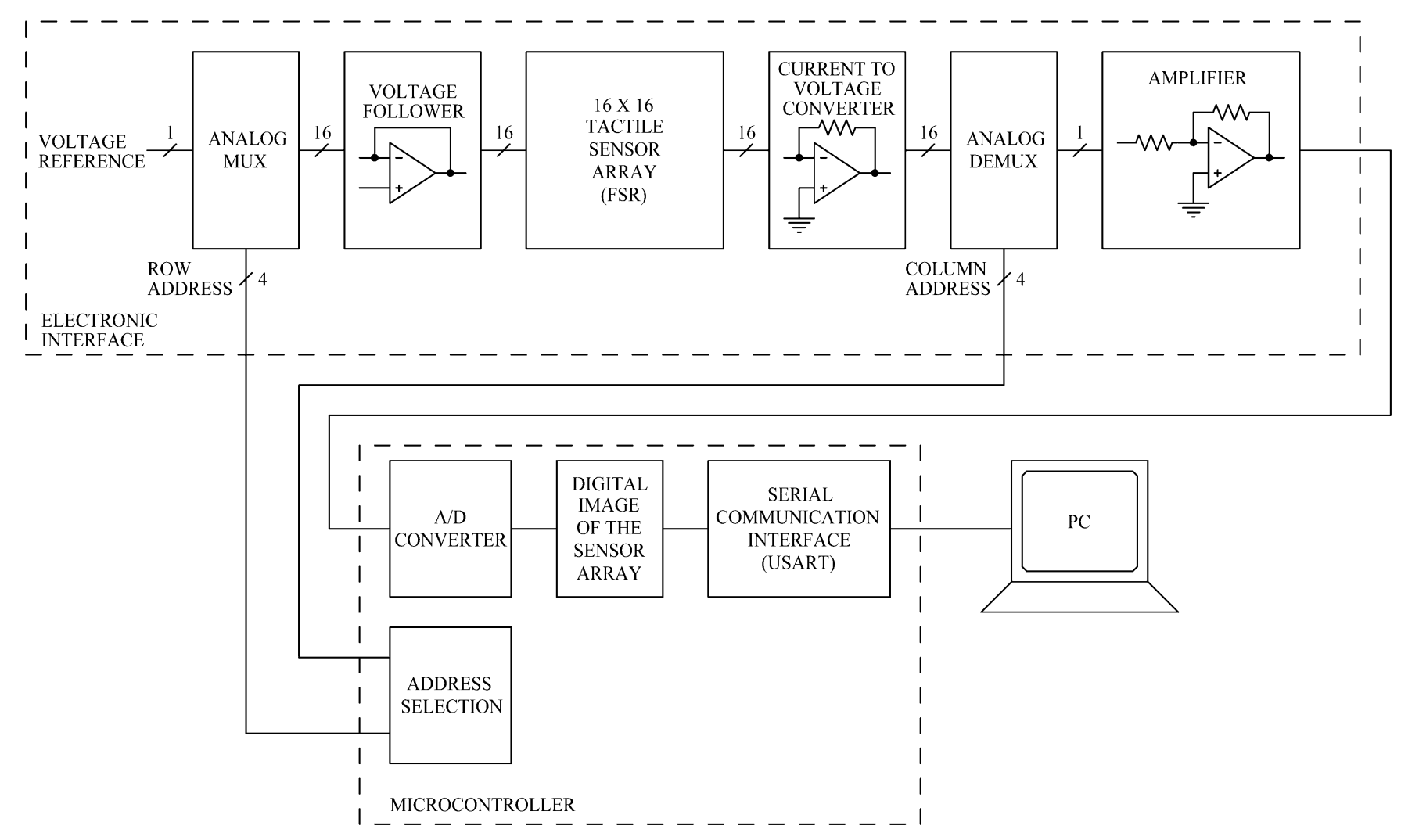

Fig. 5. Block diagram of the tactile sensor interface.

without any stress allowing for a proportional relationship between the displacement in the normal direction and the resulting stress component in each tab. As a result, the tactile probe output is a $16 \times 16$ array of data that represents normal displacement components of the 3-D geometric profile of the investigated object surface $[z(i, j) \mid i=1, \ldots, 16 ; j=1, \ldots, 16]$, where $i$ and $j$ are the row and column coordinates of the tactile sensor matrix. Fig. 3(b) presents the experimental strain/stress response of a single tab of this elastic overlay.

The typical accuracy that can be achieved with the tactile sensor prototype ranges from $5 \%$ to $25 \%$ deviation from the actual force value. While this is not sufficient for high-precision measurements, it is far enough for surface shape mapping and recognition of small objects as proposed in this paper.

\section{B. Tactile Sensor Controller and Interface}

The operation of the FSR array and the conversion from resistance values to geometric displacements for the $16 \times 16$ pixels of the tactile image is achieved by integrating the tactile sensor in a dedicated electronic circuitry using the PIC microcontroller technology. This provides full control of the sensor through a graphical user interface running on a host PC workstation. Fig. 5 illustrates the block diagram of the tactile sensor controller. Two analog multiplexer circuits, respectively, for the selection of row and column addresses, allow random access to any individual force sensitive resistor within the $16 \times 16$ FSR array. The resistance of each selected FSR element is measured by a 10-b analog/digital converter onboard the 16-b PIC microcontroller. The microcontroller also ensures transmission of data

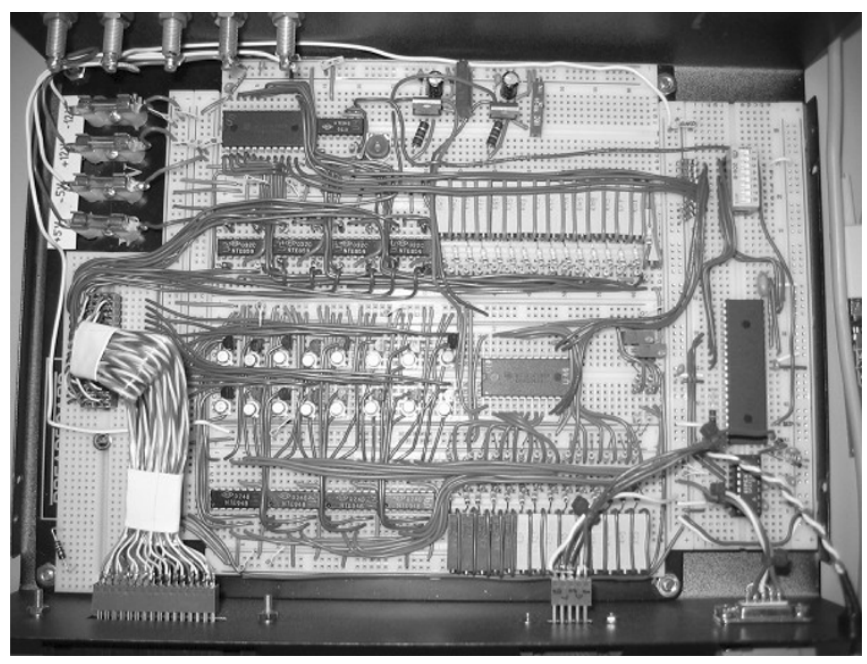

Fig. 6. Tactile sensor electronic controller.

to the graphical user interface via a standard serial communication link operating at $19200 \mathrm{~b} / \mathrm{s}$. Fig. 6 presents the hardware implementation of the first prototype of the dedicated controller interfaced with the FSR array. A more compact version is under development that will allow onboard installation on the robot arm and facilitate displacements of the sensor.

Data acquisition, download of measurements to the host PC as well as image filtering functionalities are conveniently accessible from the graphical user interface that is shown in Fig. 7. This interface has been built on Matlab 6.5 running on MS Windows 2000. Displacement measurements are scaled on a range 


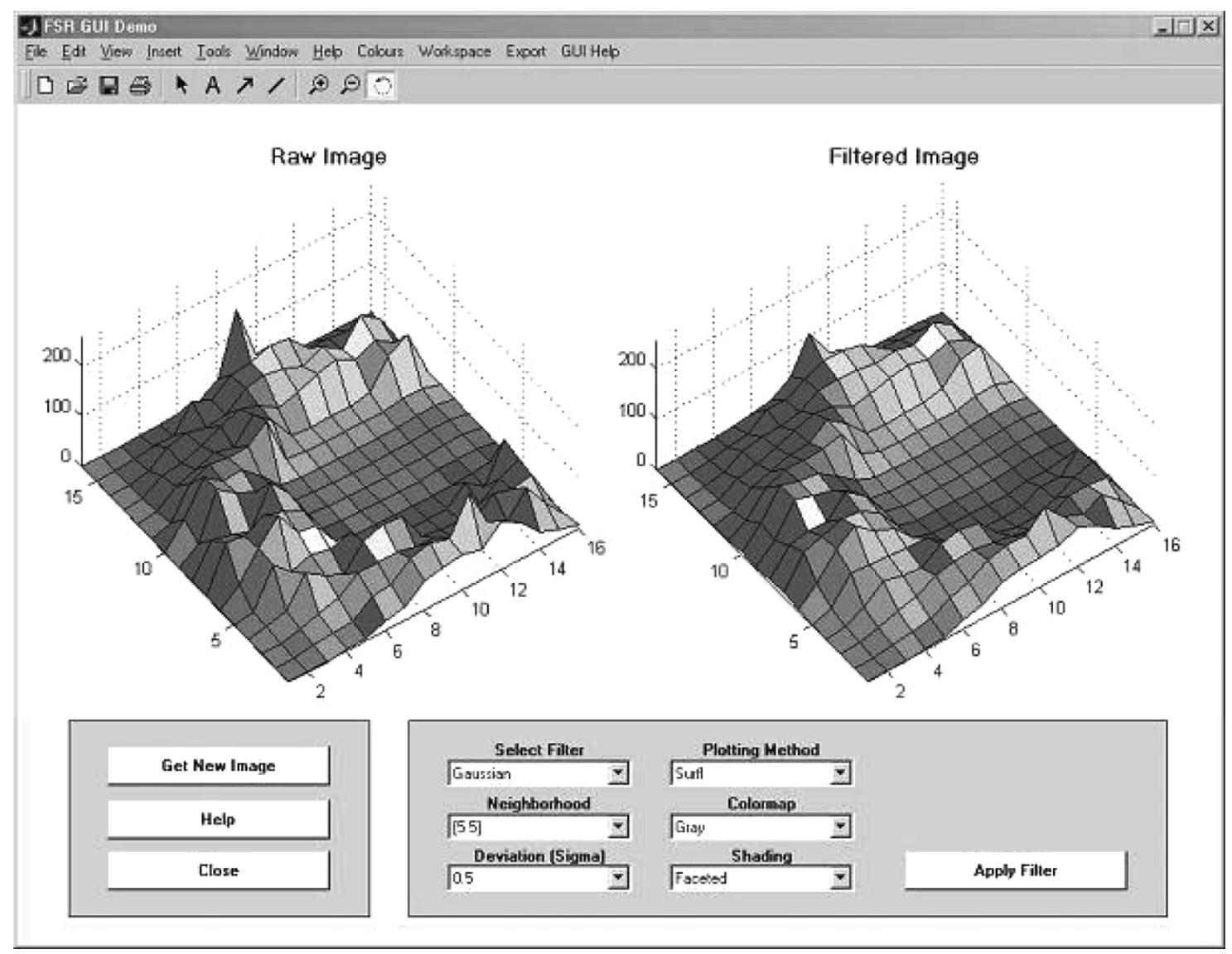

Fig. 7. Graphical user interface developed to operate the tactile sensor.

comprised between 0 and 256 and can be saved in different proprietary formats to be eventually reloaded in the interface in order to perform supplementary processing. Standard filtering operations such as averaging, mean, median, and Gaussian, all with selectable sizes of neighborhood, can also be directly performed on the raw data. These functions are extremely helpful in preprocessing data when the level of noise on measurements is significant.

\section{OBJeCt RECOGNITION With TACTILE DATA}

The intelligent haptic sensor system has initially been tested for model-based blind tactile recognition of 3-D objects. For this purpose, four conveniently shaped geometric symbols representing quaternary terms of a pseudorandom array (PRA) defined over the Galois field $\mathrm{GF}(4)=\left\{0,1, A, A^{2}\right\}$ [28] were embossed on wooden object surfaces as shown in Fig. 8. Symbols recovered by tactile probing are recognized using a neural network and then clustered in a $2 \times 2$ PRA window that contains enough information to fully identify the absolute coordinates of the recovered window within the encoded PRA. By knowing how different object models were mapped to the PRA, it is possible to unambiguously identify the object face and the exact position of the recovered symbols on the face [29].

A two-layer feedforward architecture, with eight neurons in the hidden layer and four in the second one as shown in Fig. 9, is employed to classify tactile data representing the four encoding symbols. The choice for a multilayer feedforward architecture
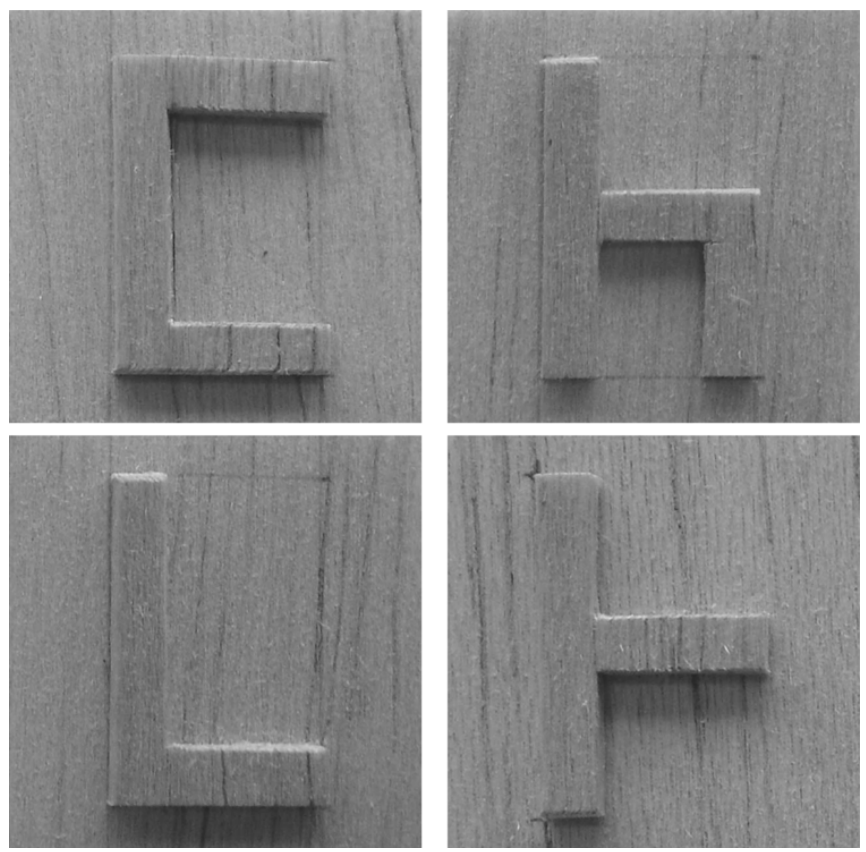

Fig. 8. Four geometric symbols tested during the design of the tactile object recognition system.

based on supervised learning is justified first by the need for supervised learning, which is known to be the proper tool for pattern recognition, and second by the fact that multilayer networks are trained using backpropagation, which is more pow- 


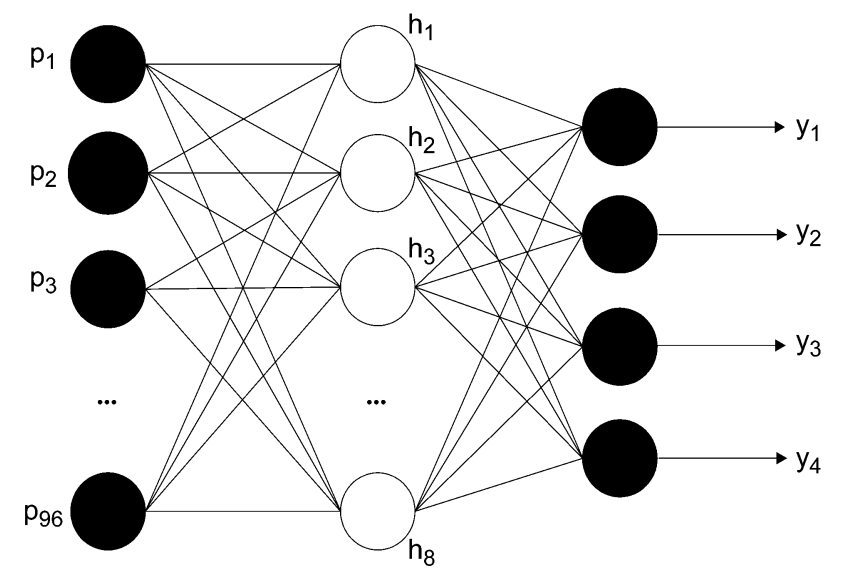

Fig. 9. Two-layer feedforward neural network architecture for four symbols classification.

erful and more flexible than all the training algorithms used by single-layer networks. Here, a very simple multilayer architecture is used, with only one hidden layer. There are four nodes chosen in the output layer in order to achieve orthogonal representations for the four different classes that are to be recognized and, therefore, ensure a better learning of the trained network.

Each $16 \times 16$ tactile image is initially truncated to an $8 \times 12$ image to keep the focus on the embossed area containing the symbol. This reduces the complexity of the neural network. The resulting 96 elements are provided as an input vector $p_{i}$ to the network and the corresponding target symbol is identified by the largest value appearing on one of the output nodes, $\left[\max \left(y_{k}\right) \mid\right.$ $k=1, \ldots, 4]$, that corresponds to the position of the recognized symbol.

The network is trained with four $8 \times 12$ binary maps of the tactile images representing the centered four symbols. A gradient descent backpropagation with momentum and adaptive learning rate is used as a training algorithm. A value of 0.95 for the momentum constant and a sum-squared-error goal of 0 are applied over 5000 epochs.

The neural network is initially trained using two noise-free tactile images of each of the symbols and then with a series of images corrupted with Gaussian noise having a standard deviation of $0.1,0.15$, and 0.2 , respectively. It is finally trained one more time with noise-free images in order to make sure that noise-free images are always identified correctly. To evaluate its generalization capability, the network was tested for different levels of Gaussian noise with a 0 mean and a standard deviation varying from 0 to 0.5 . Fig. 10 shows raw tactile images of the four symbols that are recognized by the network. The average recognition rate for each symbol is depicted in Fig. 11.

Another set of tests has been run to validate the behavior of the network for test scenarios where the symbols were not properly aligned in a vertical position. The network presented previously only allows to detect symbols rotated by steps of $90^{\circ}$. To solve this problem, the initial symbol recognition module is cascaded with a transformation module. The purpose of the latter is to recuperate the displacement information (in rotation, scaling, and translation) between a raw symbol and a set of symbols stored in a database of centered and aligned objects.
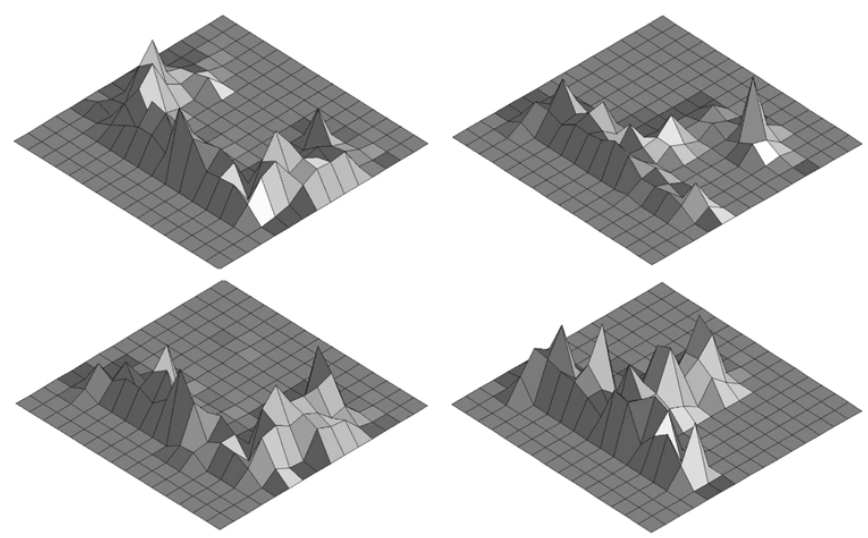

Fig. 10. Tactile images of the four symbols recognized by the neural network.

The transformation neural network has no hidden layer and receives as inputs a vector of 96 pixels $p_{i t}$ measured on the rotated symbol and as target a vector of 96 pixels $p_{i}$, corresponding to the same symbol with a correct alignment from the database. Scanning through the database containing the set of possible symbols until a suitable match is found, the network learns the displacement information and stores it in its internal weights $W$ as shown in Fig. 12. This information is then used to align the rotated symbol to the vertical position such that it can be subsequently recognized by the network shown in Fig. 9. The inverse of the weight matrix is multiplied with the transformed symbol in order to obtain the realigned 96 output pixels $p_{i a}$.

The transformation network uses a linear activation function and is trained using gradient descent backpropagation with a constant momentum value of 0.95 and an adaptive learning rate. A null sum-squared-error is targeted over 1000 training epochs. The training procedure lasts for about $5 \mathrm{~s}$.

A sample of rotated tactile data is shown in Fig. 13(a). Note that the misalignment is with respect to the pixels grid of the image. Fig. 13(b) shows the corresponding data after realignment of the tactile image with the rotation parameters estimated by the transformation neural network. The noise appearing on the realigned geometric symbol is due to the inexact learning of the neural network which not only finds rotation angles, but also scaling and translation parameters. However, this does not preempt the system from classifying the symbols correctly. As expected, once the training is completed, the raw object is aligned with the original symbol in the database, and this aligned object becomes the input of the tactile recognition module that is now able to map it to the proper symbol.

\section{EXPERIMENTATION WITH THE HAPTIC SENSOR IN INDUSTRIAL APPLICATIONS}

In order to validate its performance and pursue its development, the proposed sensing system has been mounted on a six degrees-of-freedom manipulator equipped with a laser range finder and used in modeling a 3-D scene on which a manipulator has to operate as shown in Fig. 14(a). The application considered as a testbed is that of detecting, identifying, and estimating the orientation of screw heads of different types widely found in industrial setups, such that a robot can properly position a screwdriver and remove the screws without external assistance. 


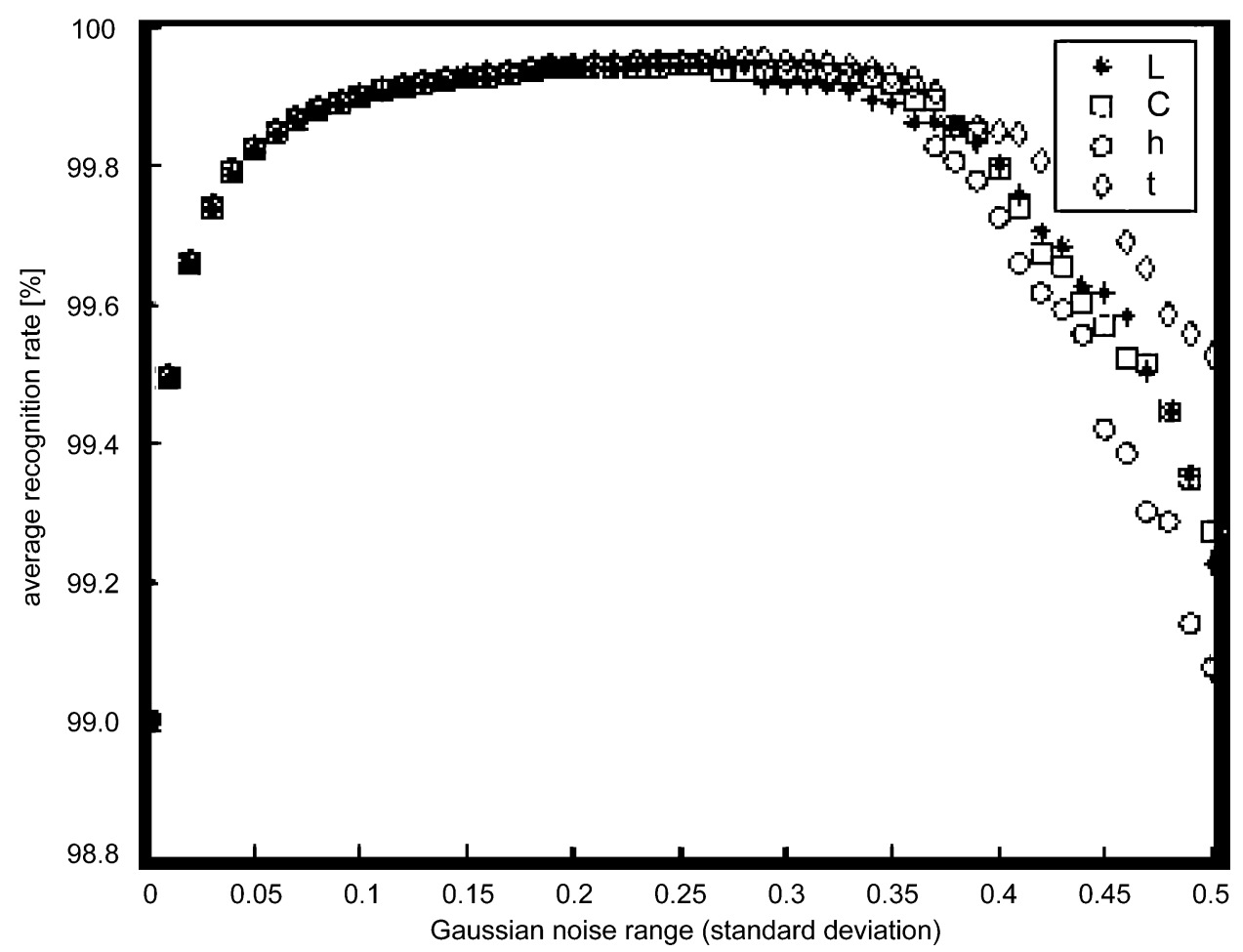

Fig. 11. Average recognition rate for tactile data with a Gaussian noise having a standard deviation ranging between 0 and 0.5 .

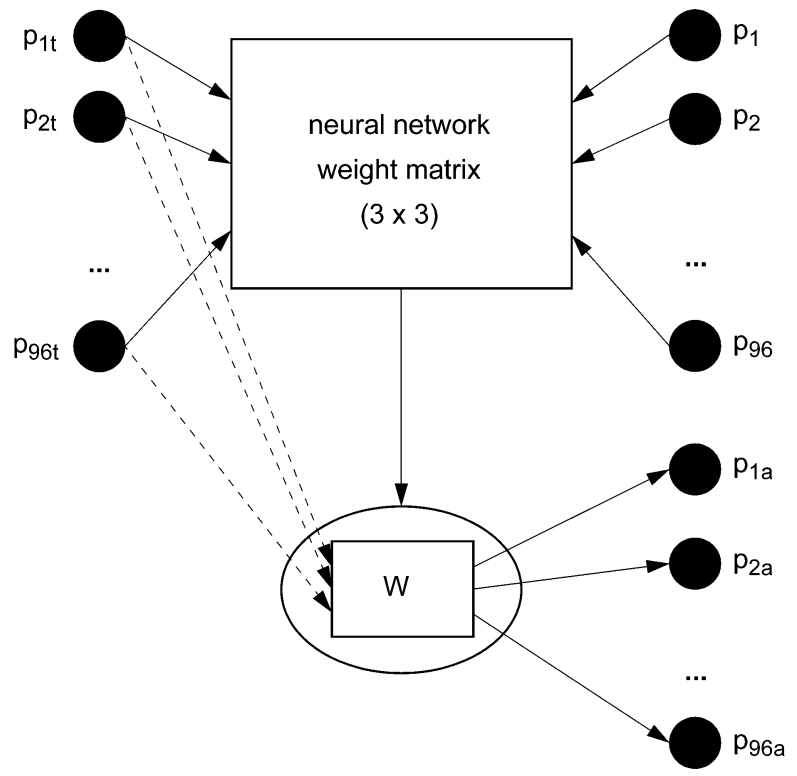

Fig. 12. Neural network architecture of the transformation module for orientation estimation.

The first step of the experimentation consisted of scanning the whole scene with the laser range finder to locate the areas occupied by the screws. Next, a refinement of the 3-D model of these regions is achieved with the haptic sensor to allow identification of the screw head type, and the required screwdriver tip, along with an estimate of the orientation of the screw slot.

\section{A. Location of Screws With a Laser Range Finder}

Even though laser scanners offer very high resolution that should theoretically allow a precise localization of tiny parts
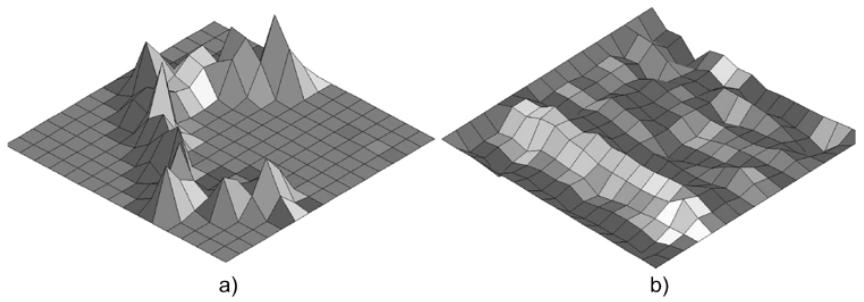

Fig. 13. (a) Raw rotated tactile data for symbol C. (b) Corresponding realigned data.

such as screws, practical experimentation demonstrates that the sensitivity to textures and reflectivity of surfaces of these sensors is too high to achieve sufficient accuracy. In this application, a Jupiter laser line scanner commercialized by Servo-Robot, Inc. [30] and built on the well-known synchronized triangulation technology developed at the National Research Council (NRC) [4] is used to locate an electrical box that has to be opened on a flat wooden panel.

Relying on the high resolution of the laser scanner that should typically reach $0.1 \mathrm{~mm}$ at a distance of $1 \mathrm{~m}$, it is expected that screw heads could be recognized by type. However, the metallic surface of the electrical box tends to act as a specular surface and generates important diffusion of the laser beam making the signal received by the sensor very noisy in this area in comparison with the wooden surface whose reflectivity is lower and returns a larger fraction of the laser beam. Fig. 14(b) shows the resulting range profile obtained with the Jupiter range finder for a scan line intersecting with one of the screws on the electrical box. It is clear from this example that even a high-end laser range scanner cannot properly handle situations where high precision is required or when operation occurs in harsh environments. In this case, the laser scanner allows a precise localization of the 


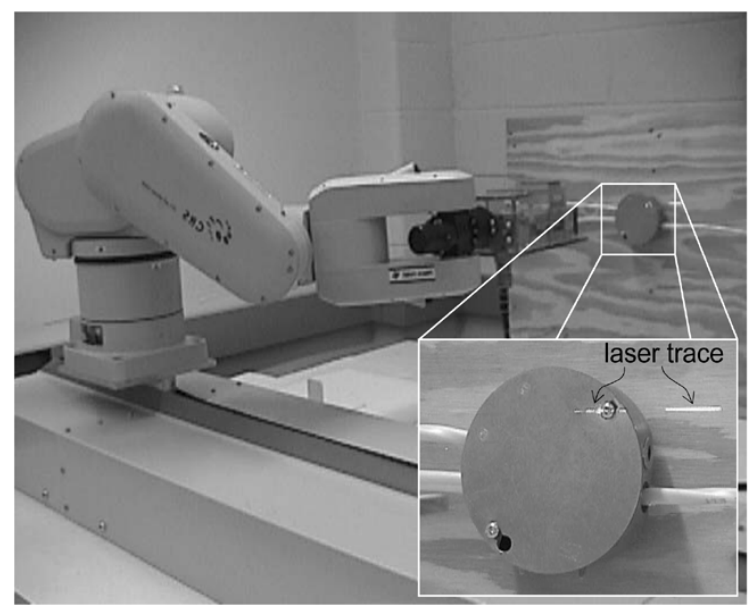

a)

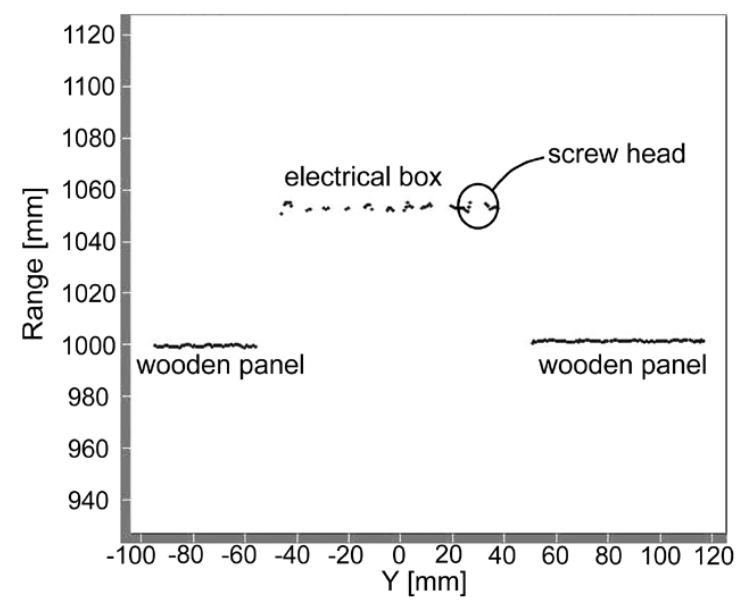

b)

Fig. 14. Experimental setup in which (a) a laser range finder is used to collect (b) a distance profile of an electrical box to guide the haptic sensor for precise location and identification of screw heads.

electrical box but hardly provides an estimate of the exact position of the screw head. It is then impossible to rely on this information only to determine the type of screw head and its orientation in order to select the proper screwdriver tip and alignment in space. Therefore, there is a need to refine the knowledge in this area of the scene by bringing the haptic sensor to collect precise measurements on the screw head.

\section{B. Refining the Model of Screws With the Haptic Sensor}

The proposed haptic sensor has then been mounted on the robot end-effector and directed in the region of the screw of interest relying on the information previously collected with the laser range scanner. The robot arm end-effector is brought in a position and orientation that makes the haptic sensor perpendicular to the cover of the electrical box. However, as the orientation of the electrical box is not defined with a very high precision, the passive-compliant wrist accommodates the differences that might remain between the orientations of the sensor and the object as shown in Fig. 15(a). The screw head is located within the 1 square inch surface of the tactile sensor as seen in Fig. 15(b).

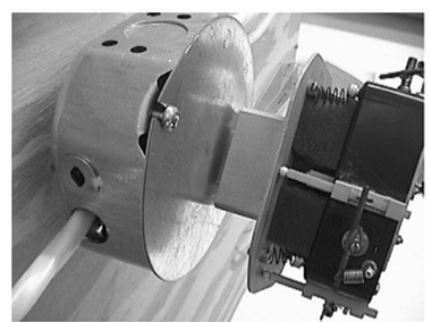

a)

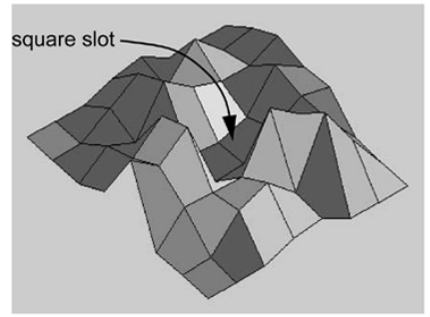

c)

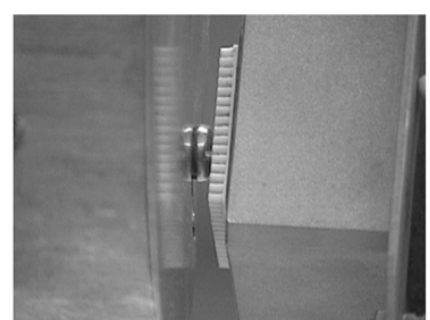

b)

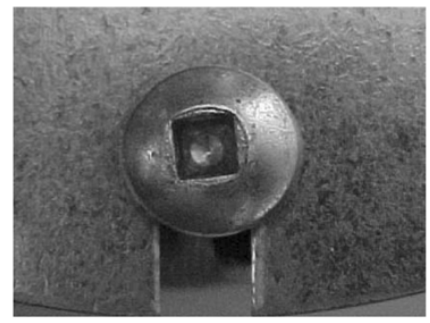

d)
Fig. 15. Measurement of the screw head position and shape with (a) the passive-compliant wrist accommodating slight misalignment and (b) the screw head located within the tactile probe area to generate (c) a tactile image of (d) a round head screw with a square slot.

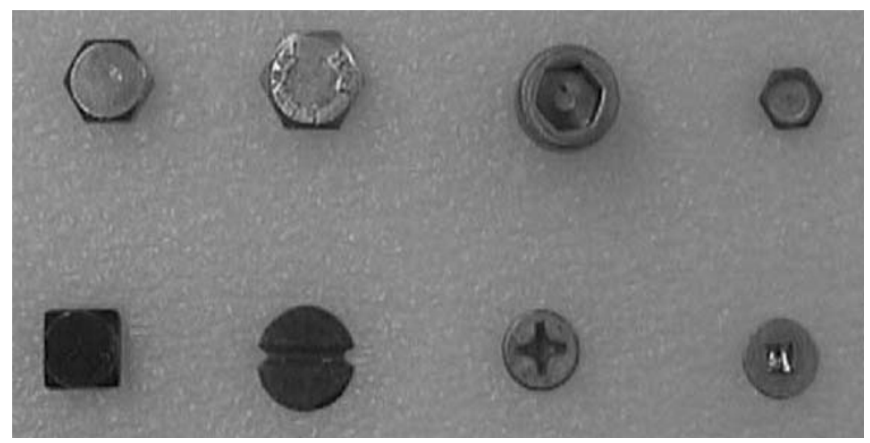

Fig. 16. Various types of screws tested for identification with the haptic sensor.

Fig. 15(c) illustrates the surface representation of the raw tactile data (not filtered) collected on the screw shown in Fig. 15(d). Considering that the maximum deflection of the tactile sensor pad that is used in this prototype is about $1 \mathrm{~mm}$, raw data provide relatively clear information about the round surface of the screw head and allow the detection of the square slot. In comparison with the precision achieved with the laser range scanner, the haptic sensor provides a significant improvement in accuracy for the location, the identification, and the evaluation of the orientation of the screw head.

\section{Generalization to Various Types of Screw Heads}

In order to improve the robustness of the robotic haptic sensing system, a generalization of the previous application has been realized to allow the recognition and orientation estimation for various types of screw heads that are found in practice. Fig. 16 presents a variety of screw heads that have been investigated using raw data collected with the proposed tactile probe.

A similar neural network as the one presented in Fig. 9 is used to identify them. This time, the input size map has been truncated to $8 \times 8$ pixels to best fit with the size of the screw heads that is about one half of a inch on average. Therefore, the neural 


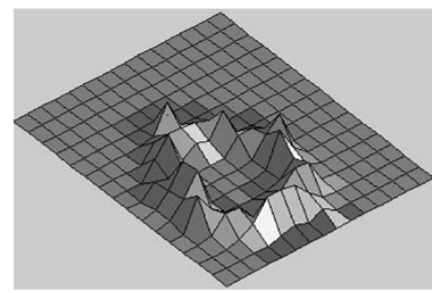

a)

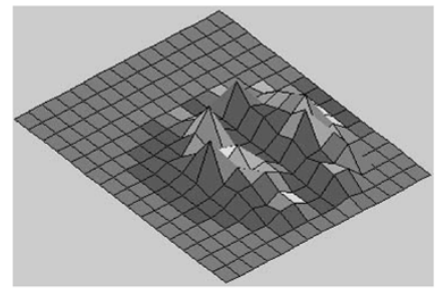

c)

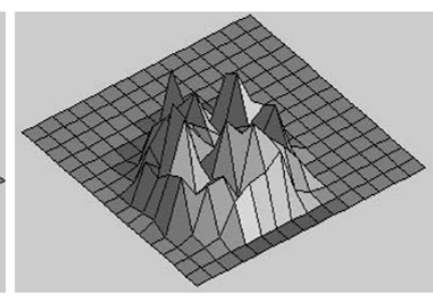

b)

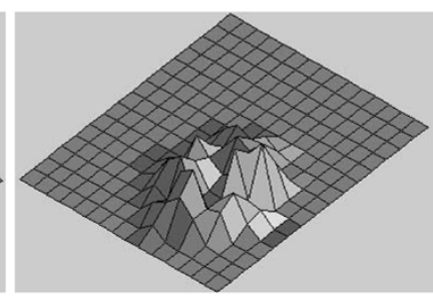

d)
Fig. 17. Sample of raw tactile data collected on four different types of screw heads: (a) hexagonal slot, (b) hexagonal head, (c) straight slot, and (d) square slot.

network used in this process only has 64 inputs, 25 neurons in the hidden layer, and a number of outputs equal to the number of screw types to be identified (eight in the presented case).

The network is trained, as in the case of symbol recognition, with two nonnoisy copies of $8 \times 8$ binary maps of screw heads, then with copies corrupted by Gaussian noise with a standard deviation level of $0.1,0.15$, and 0.2 respectively, and finally one more time with the nonnoisy copy. This procedure leads to better robustness and an enhancement in the generalization capabilities of the neural network required to compensate for the limited accuracy of the tactile pad.

After being trained for 1000 epochs, with a sum-squarederror goal of 0 , the network is able to correctly identify all the maps corresponding to screw heads in Fig. 16, apart from the one with cross embossment (Phillips type). This is due to the characteristics of the tactile pad which has tactile tabs that are close to the size of the slots on the screws. Fig. 17 presents a sample of raw tactile data collected on four different types of screws that have been classified correctly by the neural network.

For the estimation of the screw heads orientation, experiments revealed that for nonsymmetrical heads like the straight slot, the transformation module is able to distinguish between horizontal and vertical positions. For symmetrical heads like the hexagon or the square heads, orientation is less critical as the screwdriver can be positioned anywhere within the 90 degrees range without compromising the robotic task. The resolution on the estimation of the orientation is mainly limited by the size of the tactile probe tabs that do not allow finer measurements of the edges of the screw slots. This limitation is expected to be easily overcome with the development of a higher accuracy tactile sensor with smaller tabs. The neural network architectures developed for the present application will remain perfectly valid as they do not depend on the physical size of pixels in the tactile image. In terms of performance, the computational time for classification of the object is of the order of seconds when running on Matlab. It is definitely not the limiting factor in the type of application considered as positioning the robot arm, and the tactile probe appears to be much slower due to the robot's servo loop response time.

\section{CONCLUSION}

An original combination of sensing devices and neural network architectures has been developed to enhance the capabilities of robotic systems for intervention on small devices in industrial applications. An innovative haptic sensing system composed of a passive-compliant wrist and a robust tactile probe with a relatively high resolution of $1 / 16$ in is presented along with its controller and interface modules. The passive-compliant wrist component of the sensor provides accommodation for slight misalignment of the robotic arm in delicate manipulation operations while ensuring that the registration of tactile data is kept accurate.

Using neural network technologies, tactile data processing stages have been developed to allow recognition and geometric transformation estimation on a set of geometric symbols encoding a pseudorandom array that can be used to locate complex objects in an unknown environment. Simulation and experimental results have demonstrated that the neural network recognition of tactile images can reach error rates lower than $0.6 \%$ even in the case of images having up to a $50 \%$ noise ratio.

Experimentation has also been conducted on a realistic setup where the tactile probe helps to overcome the physical limitations of a commercial laser range finder in locating and determining the orientation of screw heads to be removed by a robot without external intervention. Excellent results were obtained using this first prototype of the haptic sensor.

Ongoing research work includes the development of a more compact controller that will be mounted directly on the robot arm to provide increased flexibility and make possible the straightforward migration of the calibration and basic image filtering functions, which are still running on the PC. The design of a higher resolution tactile sensor is also considered to allow robotic systems to deal with smaller objects while reducing the level of noise. Finally, automation of the multimodal sensing system is being investigated to allow automatic switching between the laser range and the haptic sensor such that an integration of tactile surface maps with laser range data can be achieved efficiently.

\section{REFERENCES}

[1] S. F. El-Hakim, J.-A Beraldin, G. Godin, and P. Boulanger, "Two 3-D sensors for environment modeling and virtual reality: Calibration and multiview registration," Int. Arch. Photogrammetry Remote Sens., vol. 31, pp. 140-146, 1996.

[2] R. Y. Tsai, "A versatile camera calibration technique for high-accuracy 3-D machine vision metrology using off-the shelf TV cameras and lenses," IEEE J. Robot. Autom., vol. RA-3, no. 4, pp. 323-344, Aug. 1987.

[3] L. G. Shapiro and G. C. Stockman, Computer Vision. Englewood Cliffs, NJ: Prentice-Hall, 2001.

[4] M. Rioux, "Laser rangefinder based on synchronized scanning," Appl. Opt., vol. 23, pp. 3837-3844, 1985.

[5] E. Trucco and A. Verri, Introductory Techniques for 3-D Computer Vision. Englewoods Cliffs, NJ: Prentice-Hall, 1998.

[6] G. Canepa, O. Sottile, and D. De Rossi, "Extraction of cutaneous primitives from tactile sensor images," in Proc. IEEE Int. Conf. Systems, Man and Cybernetics, vol. 3, 1994, pp. 2641-2646.

[7] R. D. Howe, W. Peine, D. A. Kontarinis, and J. S. Son, "Remote palpation technology," IEEE Eng. Med. Biol. Mag., vol. 14, no. 3, pp. 318-323, May/Jun. 1998.

[8] H. Tobita, T. Kawamure, Y. Sugimoto, and H. Nakamura, "The development of 'safe partner equipment',' in Proc. IEEE Int. Conf. Robotics and Automation, Nagoya, Japan, 1995, pp. 2420-2426. 
[9] T. Itoh, K. Kosuge, and T. Fukuda, "Human-Machine cooperative telemanipulation with motion and force scaling using task-oriented virtual tool dynamics," IEEE Trans. Robot. Autom., vol. 16, no. 5, pp. 505-516, Oct. 2000.

[10] R. D. Howe and M. R. Cutkosky, "Dynamic tactile sensing-Perception of fine surface-features with stress rate sensing," IEEE Trans. Robot. Autom., vol. 9, no. 2, pp. 140-151, Apr. 1993.

[11] D. Johnston, P. Zhang, J. Hollerbach, and S. Jacobsen, "A full tactile sensing suite for dexterous robot hands and use in contact force control," in Proc. IEEE Int. Conf. Robotics and Automation, Minneapolis, MN, 1996, pp. 3222-3227.

[12] H. Zhan and N. N. Chen, "Control of contact via tactile sensing," IEEE Trans. Robot. Autom., vol. 16, no. 5, pp. 482-495, Oct. 2000.

[13] F. Germagnoli and G. Magenes, "A neural network-based system for tactile exploratory tasks," in Proc. Int. Workshop Neural Networks for Identification, Control, Robotics, and Signal/Image Processing, 1996, pp. $458-466$.

[14] J. L. Pedreno-Molina, A. Guerrero-Gonzalez, and J. Lopez-Coronado, "A neural controller for a robotic hand with artificial tactile skins in grasping tasks," in Proc. IEEE Int. Conf. Systems, Man and Cybernetics, vol. 1, Apr. 2000, pp. 161-165.

[15] D. Taddeucci, C. Laschi, R. Lazzarini, R. Magni, P. Dario, and A. Starita, "An approach to integrated tactile perception," in Proc. IEEE Int. Conf. Robotics and Automation, Albuquerque, NM, 1997, pp. 3100-3105.

[16] T. Fischer, D. Rapela, and H. Woern, "Joint controller for the objectpose controlling on multifinger grippers," in Proc. Int. Conf. Advanced Intelligent Mechatronics, 1999, pp. 422-427.

[17] M. H. Lee and H. R. Nicholls, "Tactile sensing for mechatronics-A state of the art survey," in Mechatronics. New York: Pergamon, 1999, pp. $1-31$.

[18] C. S. Tzafestas, "Whole-hand kinesthetic feedback and haptic perception in dexterous virtual manipulation," IEEE Trans. Syst., Man, Cybern. A, Syst., Humans, vol. 33, no. 1., pp. 100-113, Jan. 2003.

[19] S. J. Lederman, R. L. Klatzky, and D. T. Pawluk, "Lessons from the study of biological touch for robotic haptic sensing," in Advanced Tactile Sensing for Robotics, H. R. Nicholls, Ed. Singapore: World Scientific, 1992.

[20] F. Castelli, "An integrated tactile-thermal robot sensor with capacitive tactile array," IEEE Trans. Ind. Appl., vol. 38, no. 1, pp. 85-90, Jan./Feb. 2002.

[21] E. Petriu, W. S. McMath, S. K. Yeung, and N. Trif, "Active tactile perception of object surface geometric profiles," IEEE Trans. Instrum. Meas., vol. 41, no. 1, pp. 87-92, Feb. 1992.

[22] R. Yang and P. S. Krishnaprasad, "Tactile sensing and inverse problems," Elect. Eng. Dept. and System Res. Center, Univ. Maryland, College Park, MD, 1988.

[23] R. E. Saad, A. Bonen, K. C. Smith, and B. Benhabib, "Distributed-force recovery for a planar photoelastic tactile sensor," IEEE Trans. Instrum. Meas., vol. 45, no. 2, pp. 541-546, Apr. 1996.

[24] T. H. Speeter, "A tactile sensing system for robotic manipulation," Int. J. Robot. Res., vol. 9, no. 6, pp. 25-36, 1990.

[25] Y. C. Pati, P. S. Krisnaprasad, and M. C. Peckerar, "An analog neural network solution to the inverse problem of early taction," IEEE Trans. Robot. Autom., vol. 8, no. 2, pp. 196-212, Apr. 1992.

[26] R. Q. Yang and M. W. Siegel, "A finger-tip optical sensor array sorting system,” in Proc. Sensors Conf., 1995, CASA/SME Tech. Paper MS85991, pp. 1-11.

[27] A. W. De Groot, "Effect of sensor size in robotic tactile sensor arrays," Robotica, vol. 6, pp. 285-287, 1988.

[28] E. M. Petriu, T. Bieseman, N. Trif, W. S. McMath, and W. S. Yeung, "Visual object recognition using pseudorandom grid encoding," in Proc. IEEE/RSJ Int. Conf. Intelligent Robots and Systems, vol. 3, 1992, pp. 1617-1624.

[29] E. M. Petriu, S. K. S. Yeung, S. R. Das, and H. J. W. Spoelder, "Robotic tactile recognition of pseudorandom encoded objects," in Proc. IEEE Int. Conf. Instrumentation and Measurement, Vail, CO, 2003, pp. 1397-1401.

[30] Jupiter 3-D Laser Vision Camera Installation and Operation Manual, Servo-Robot Inc., St-Bruno, QC, Canada, 1996.

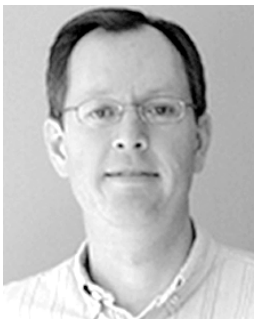

Pierre Payeur (S'90-M'98) received the Ph.D. degree in electrical engineering from Laval University, Quebec, QC, Canada, in 1999.

In 1998, he joined the University of Ottawa, Ottawa, ON, Canada, as an Assistant Professor in the School of Information Technology and Engineering (SITE) and cofounded the Vision, Imaging, Video, and Autonomous Systems Research Laboratory (VIVA). His current research interests are volumetric 3-D modeling, range data processing, robot guidance, teleoperation, and integration of computer vision in autonomous systems control.

Dr. Payeur is a Member of the IEEE Robotics and Automation Society, the IEEE Instrumentation and Measurement Society, and the Ordre des Ingénieurs du Québec.

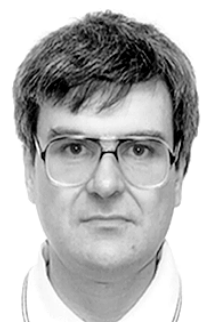

Codrin Pasca (S'04-M'05) received the B.Eng. degree in electronics and telecommunications from the University Politehnica, Bucharest, Romania, in 1988, and the M.A.Sc. degree in electrical engineering from the University of Ottawa, Ottawa, ON, Canada, in 2004.

He is currently a Research Assistant in the Sensing and Modeling Research Laboratory, School of Information Technology and Engineering, University of Ottawa. His research interests include intelligent sensors, smart robots, and real-time embedded software.

Mr. Pasca was the recipient of the Ontario Graduate Scholarship for the 2003-2004 academic year.

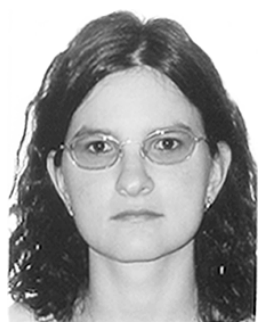

Ana-Maria Cretu (S'04) received the M.A.Sc. degree from the School of Information Technology and Engineering, University of Ottawa, Ottawa, ON, Canada, where she is currently pursuing the Ph.D.degree.

Her research interests include neural networks, tactile sensing, 3-D object modeling, and multisensor data fusion.

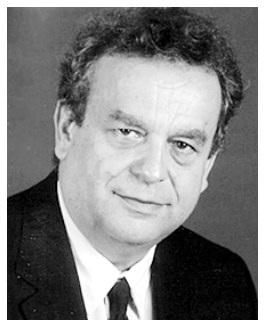

Emil M. Petriu (M'86-SM'88-F'01) is a Professor and University Research Chair in the School of Information Technology and Engineering at the University of Ottawa, Ottawa, ON, Canada. His research interests include robot sensing and perception, intelligent sensors, interactive virtual environments, soft computing, and digital integrated circuit testing. During his career, he has published more than 200 technical papers, authored two books, edited two other books, and received two patents.

Dr. Petriu is a Fellow of the Canadian Academy of Engineering and of the Engineering Institute of Canada. He is a co-recipient of the IEEE's Donald G. Fink Prize Paper Award for 2003 and recipient of the 2003 IEEE Instrumentation and Measurement Society Award. He is currently serving as Chair of TC-15 Virtual Systems and Co-Chair of TC-28 Instrumentation and Measurement for Robotics and Automation and TC-30 Security and Contraband Detection of the IEEE Instrumentation and Measurement Society. $\mathrm{He}$ is an Associate Editor of the IEEE TRANSACTIONS ON INSTRUMENTATION AND MEASUREMENT and a Member of the Editorial Board of the IEEE Instrumentation and Measurement Magazine. 